\title{
Passive Aeroelastic Tailored Wing Modal Test Using the Fixed Base Correction Method
}

\author{
International Modal Analysis Conference, IMAC XXXVIII \\ February 10-13, 2020 - Houston, TX
}

\section{Presenter: Natalie Spivey}

Feb. 13 $3^{\text {th }}, 2020$ in Session \#78, Boundary Condition Correction in Modal Testing

Authors: Natalie Spivey1, Rachel Saltzman ${ }^{1}$, Carol Wieseman², Kevin Napolitano ${ }^{3}$ \& Benjamin Smith

${ }^{1}$ NASA Armstrong Flight Research Center

2 NASA Langley Research Center

${ }^{3}$ ATA Engineering, Inc.

${ }^{4}$ Aurora Flight Sciences 


\section{PAT Wing Ground Vibration Test (GVT) - Outline}

- Fixed Based Correction (FBC) Method

- Motivation

- Theory

- Goal, Objective \& Success Criteria

- Test Article Description

- PAT Wing GVT

- Test Setup

- Test Configurations

- GVT Instrumentation

- Accelerometer Layout

- Shaker Layout for FBC

- Results

- Summary

Passive Aeroelastic Tailored (PAT) Wing GVT using Fixed Base Correction Method - July 2018

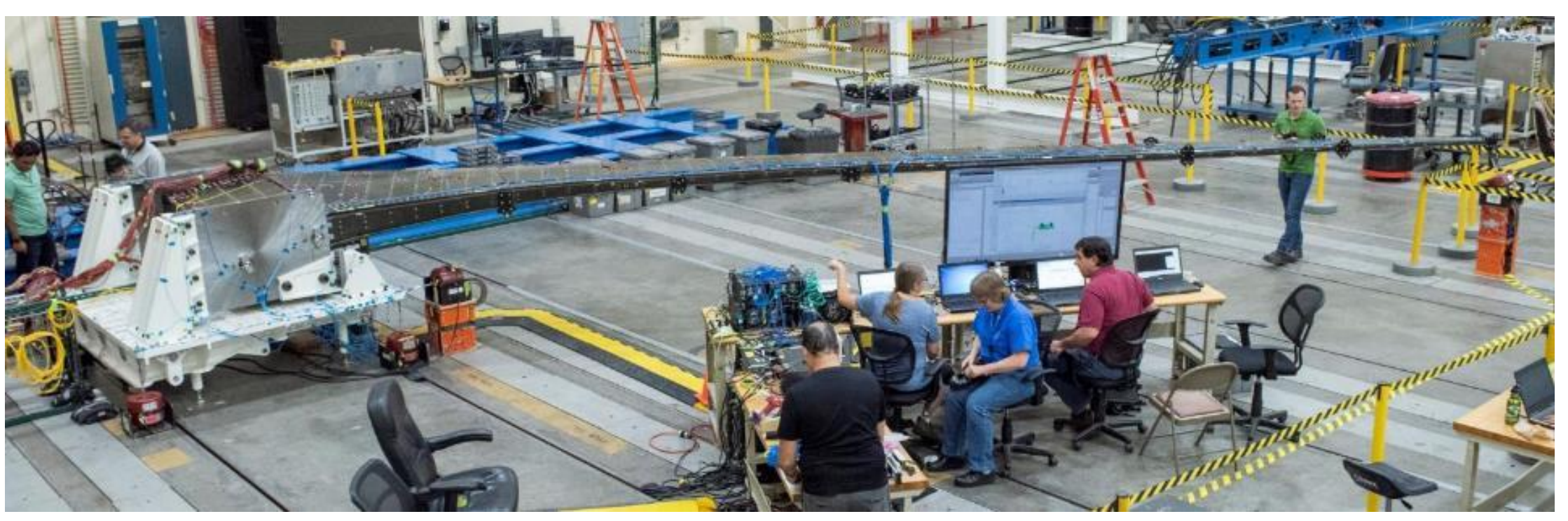




\section{Fixed Base Correction (FBC) Method - Motivation}

- Modal testing \& finite element model (FEM) correlation desire free-free or rigid boundary conditions (BC) for comparisons

- Expensive in cost \& schedule to build \& test with BC that replicate free-free or rigid

- Static test fixtures are large, heavy \& unyielding, but do not provide adequate BC for modal tests

- Dynamically too flexible \& frequencies within test article frequency range of interest

- Dynamic coupling between test article \& test fixture causes significant FEM effort

- If modal test results could be corrected for fixture coupling, then other structural testing setups may be adequate for modal testing

- Would allow significant cost \& schedule savings by eliminating a unique setup for modal testing

- NASA Armstrong evaluated the Fixed base correction (FBC) method with two recent tests

- CReW modal test was a pathfinder test to investigate FBC method prior to PAT Wing GVT where wing was cantilevered from a static test fixture with the wingtip $\approx 10 \mathrm{ft}$ off the ground

- To simplify PAT Wing GVT, the FBC method was implemented with wing cantilevered from a static test fixture on the lab floor 


\section{Fixed Base Correction Method - Theory}

- Two approaches for extracting fixed base modes from structures mounted on flexible tables

1. Constraint equation to measure mass-normalized mode shapes to generate fixed base modes

- Method requires well-excited modes so that modal mass can be accurately calculated

- Advantage - Large number of shakers do not necessarily need to be mounted on the base

- Disadvantage - Accuracy is reduced if the fixed base modes are not a linear combination of the measured mode shapes

2. FBC method uses base accelerations as references to calculate frequency response functions (FRFs) associated with a fixed base, then FRFs are analyzed to extract fixed based modes of the test article

- Fixed Base Correction GVT methodology developed by ATA Engineering, Inc. \& implemented in ATA's IMAT (Interface between MATLAB, Analysis and Test) software

- Requires multiple shakers on both the test article \& mounting fixture

- Method excites static test fixture base directly \& uses drive point accelerations as references when calculating FRFs instead of traditional shaker forces as references

- Essentially removes the fixture response from the wing response 


\section{Fixed Base Correction Method - Theory}

- FBC method can be illustrated with a simple spring-mass two degree-of-freedom (DOF) system

- Applying Newton's second law, the equation of motion for an undamped system in the frequency domain

$$
\left[\begin{array}{cc}
-\omega^{2} m_{1}+k & -k \\
-k & -\omega^{2} m_{2}+2 k
\end{array}\right]\left\{\begin{array}{l}
x_{1} \\
x_{2}
\end{array}\right\}=\left\{\begin{array}{l}
f_{1} \\
f_{2}
\end{array}\right\}
$$

- Traditional modal testing calculates FRFs using DOFs 1 \& 2 forces applied as references for the full system response

$$
a_{1}=\left[\frac{-\omega^{2}\left(-\omega^{2} m_{2}+2 k\right)}{\left(-\omega^{2} m_{2}+2 k\right)\left(-\omega^{2} m_{1}+k\right)-k^{2}} \frac{-\omega^{2} k}{\left(-\omega^{2} m_{2}+2 k\right)\left(-\omega^{2} m_{1}+k\right)-k^{2}}\right]\left\{\begin{array}{l}
f_{1} \\
f_{2}
\end{array}\right\}
$$

Spring-Mass Two DOF System

- FBC method uses DOF 1 force \& DOF 2 acceleration as references, then resulting FRFs are associated with a structural system with dynamics associated with DOF 2 fixed

$$
a_{1}=\left[\begin{array}{ll}
\frac{-\omega^{2}}{-\omega^{2} m_{1}+k} & \frac{k}{-\omega^{2} m_{1}+k}
\end{array}\right]\left\{\begin{array}{l}
f_{1} \\
a_{2}
\end{array}\right\}
$$

- FRF associated with DOF 1 applied force is equivalent to the FRF of a fixed base system

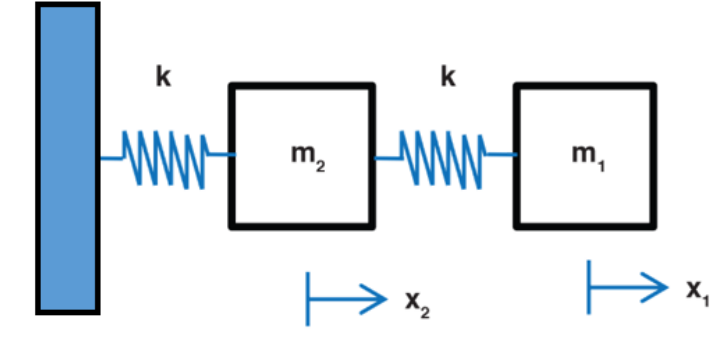

Where:

$m=$ mass

$\omega=$ frequency

$k=$ structural stiffness

$x=$ displacement

$f=$ external force

$a=$ acceleration

Subscripts $1 \& 2$ refer

to blocks $1 \& 2$

- Best practice for implementing FBC method

- Need at least one independent excitation source (i.e. shakers) for each DOF that is desired to be fixed

- Requires multiple shakers used on both test article \& test fixture (drive the base or test fixture shakers with harder forces)

- Use shaker accelerations as references rather than traditional shaker forces when calculating FRFs

- Make sure drive point FRF are as co-located as practicable \& as clean as practicable

- Use seismic accelerometers as drive points on the base 


\section{PAT Wing GVT - Goal, Objective \& Success Criteria}

- Passive Aeroelastic Tailored (PAT) Wing Ground Vibration Test (GVT) was tested July 10-12 th 2018 in NASA Armstrong's Flight Loads Laboratory (FLL)

- Goal: Obtain PAT Wing modal characteristics from the GVT to compare test results with analytical models

PAT Wing GVT - July 2018

- Objective: Measure the primary frequencies, mode shapes \& damping (frequencies up to wing torsion mode, $\approx 55 \mathrm{~Hz}$ ) using traditional accelerometers with the PAT Wing installed on the Wing Loads Test Fixture (WLTF) table

- Success Criteria: Accurately obtaining the primary frequencies and shape modes of the PAT Wing (de-coupled from the WLTF table \& attachment hardware modes) using the Fixed Base Correction (FBC) method
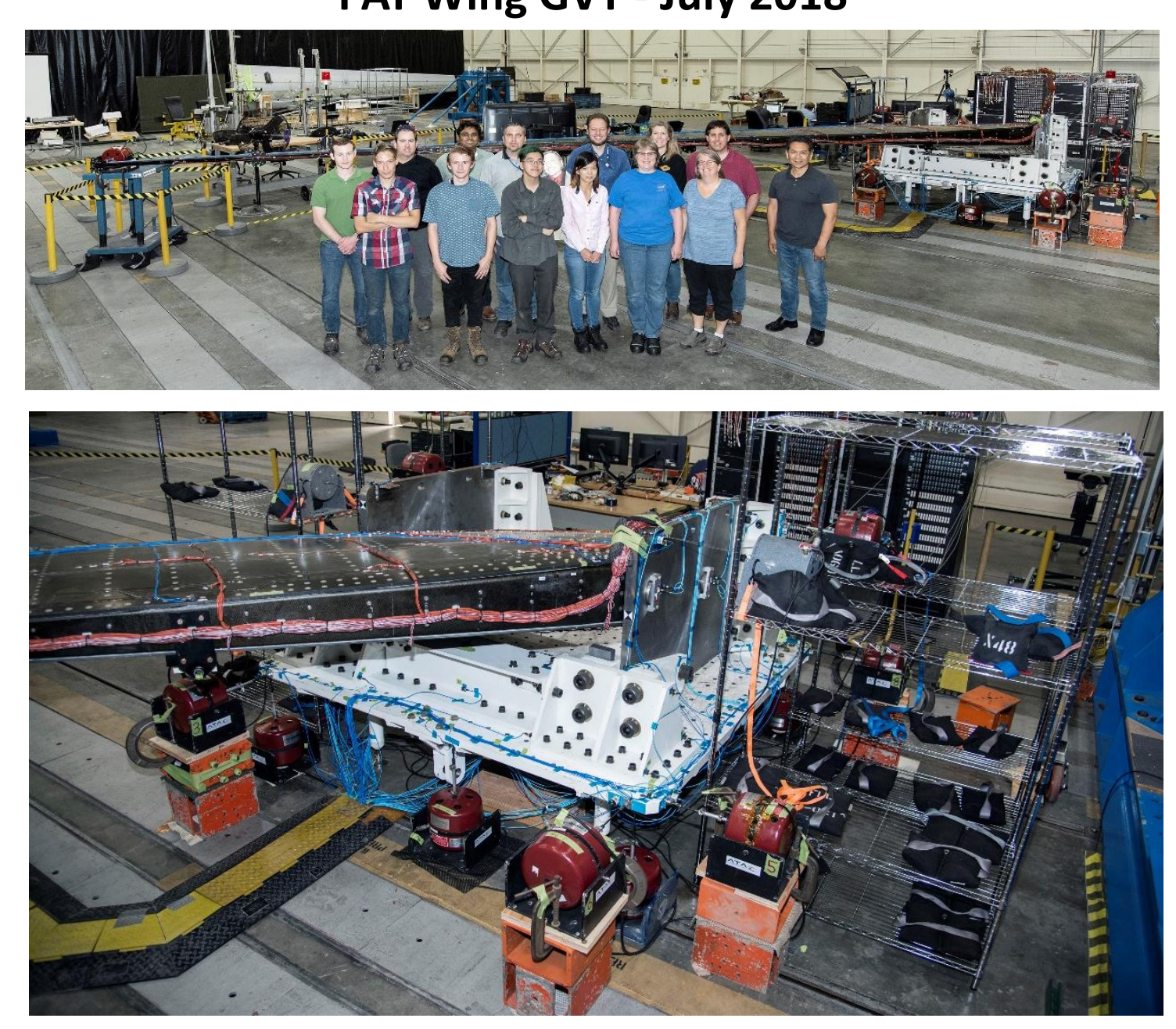


\section{Passive Aeroelastic Tailored (PAT) Wing}

- NASA's Advanced Air Transport Technology (AATT) Project desires to develop technologies to design, build \& test higher aspect ratio wings for lower induced drag and thus lower fuel burn

- Passive aeroelastic tailored structural design has been evaluating aeroelastically tailored wing structures to increase wing aspect ratio (from 9 to 14 ) and reduce weight by $20-25 \%$ without impacting aeroelastic performance

- PAT Wing Project

- Project team: Aurora Flight Sciences Corporation, NASA Langley Research Center \& NASA Armstrong Flight Research Center

- Goals

- Design \& fabricate a passive aeroelastic tailored structural wingbox using the towed-steering technology

- Create finite element models with the towed-steering technology \& conduct structural analyses

- Conduct structural ground tests to validate analytical models \& assumptions

- Ground Vibration Test - validate wing's frequencies \& mode shapes

- Flexural Axis Test - validate wing's bend twist coupling response

- Static Load Test - validate wing's response including stiffness, strains \& deformations

Passive Aeroelastic Tailored (PAT) Wing

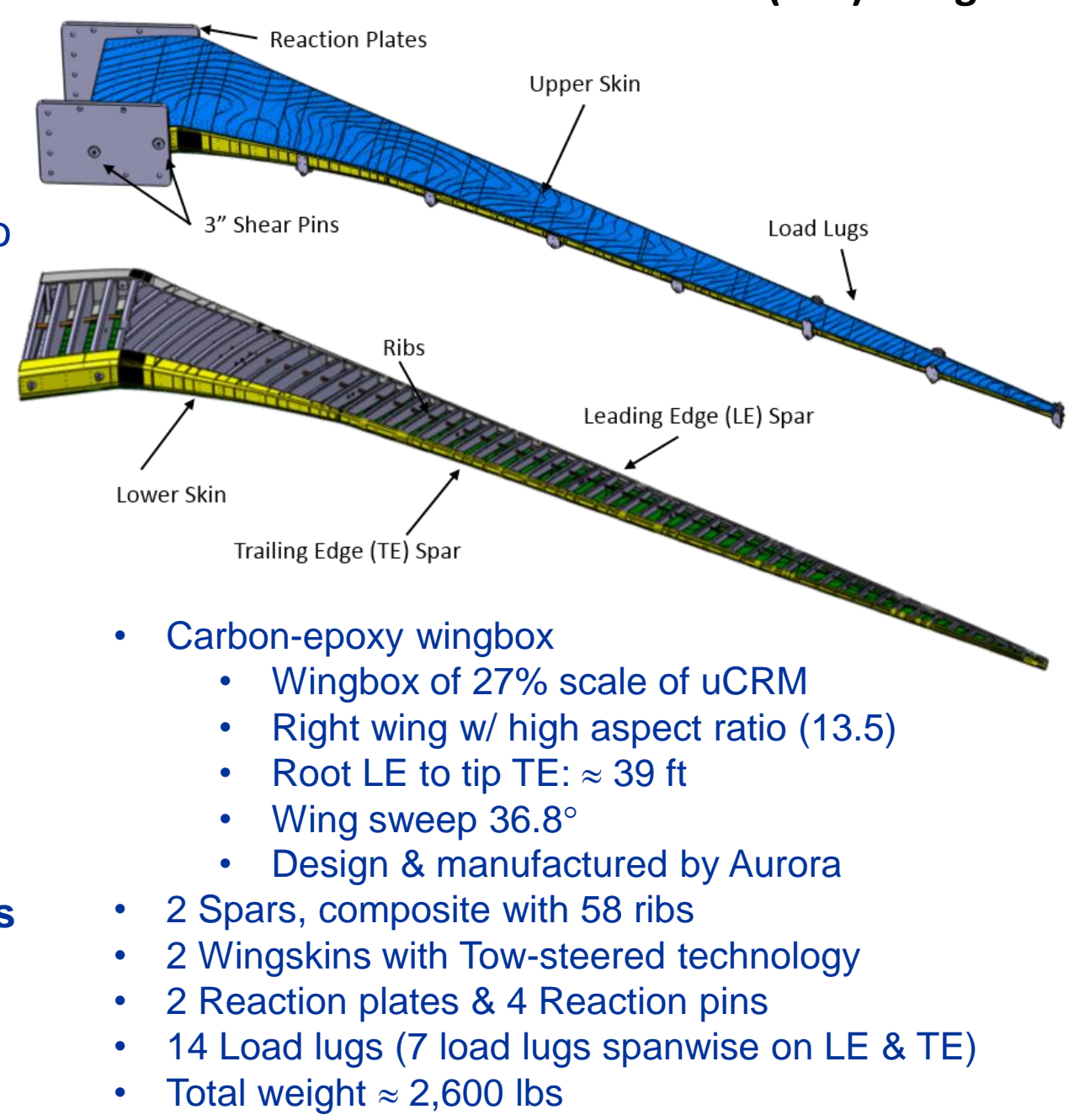




\section{Test Setup - GVT Test Setup, Original Plan}

- Original plan: Perform GVT using Fixed Base Correction on the Wing Loads Test Fixture (WLTF) to save cost and schedule rather than different boundary conditions from the loads testing

- Reaction plates mounted with attachment hardware to WLTF table

- Wingtip $\approx 124$ " off ground

- Overhead loading structure installed

\section{Overhead Loading}

Structure

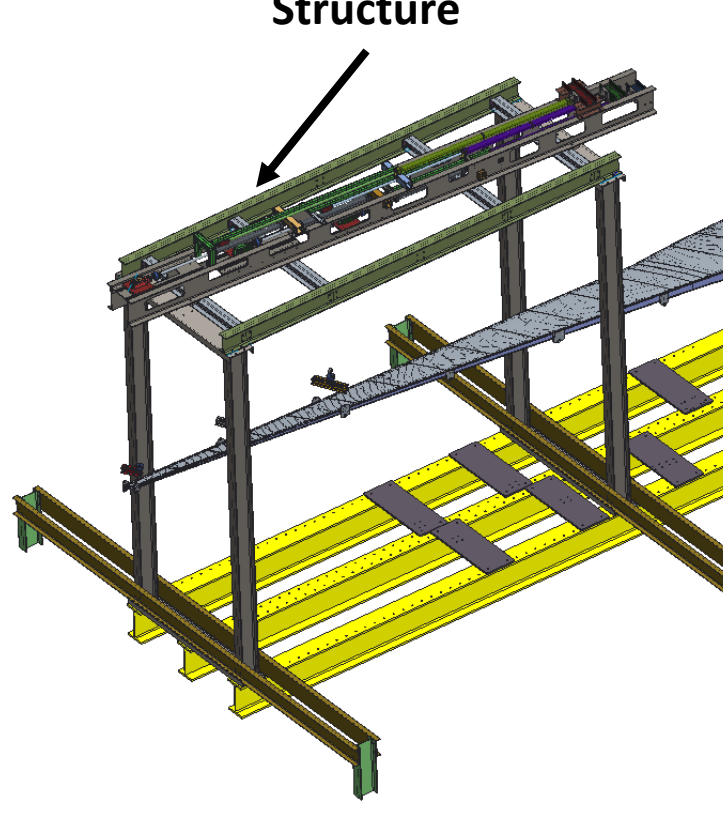

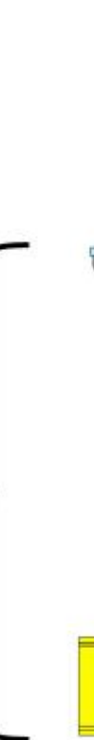

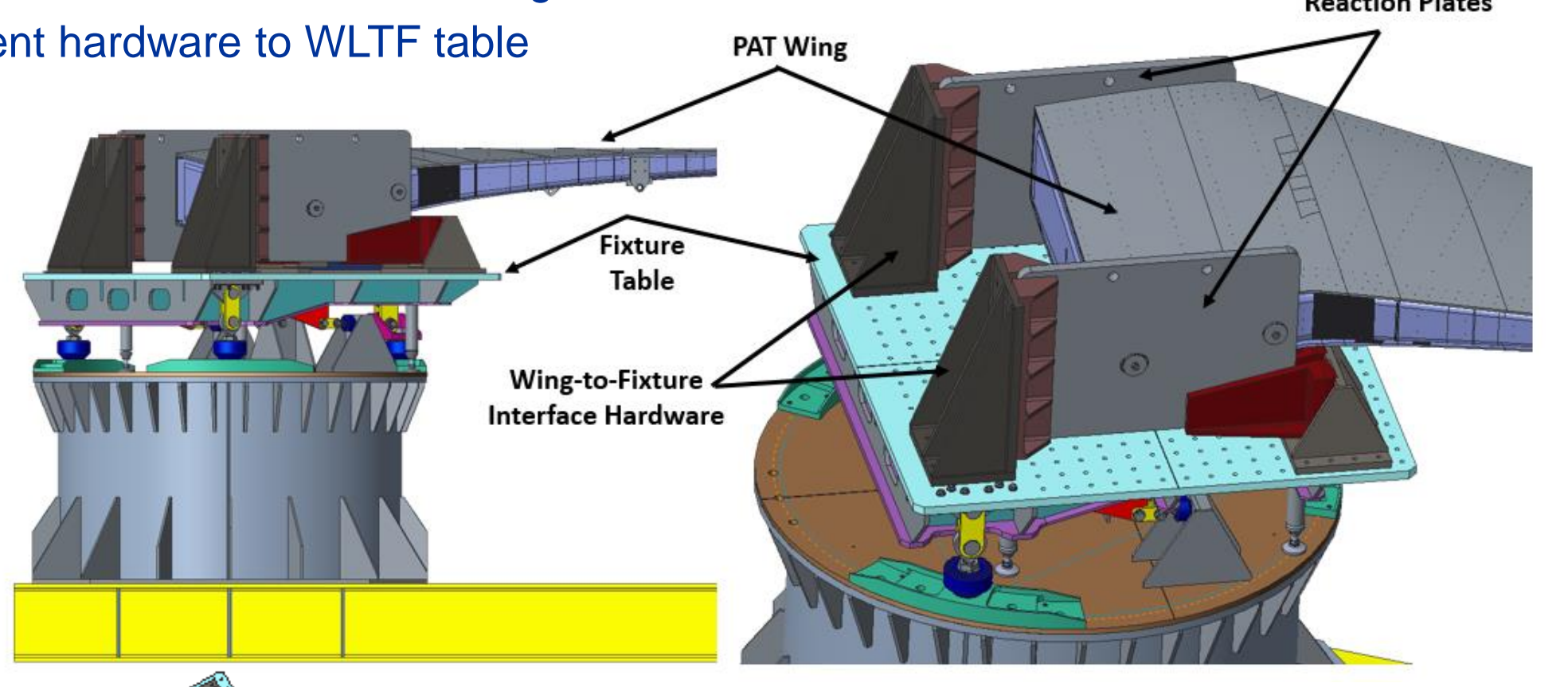

Reaction Table

(rotated $30^{\circ}$ )

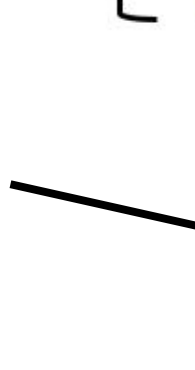

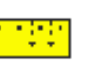

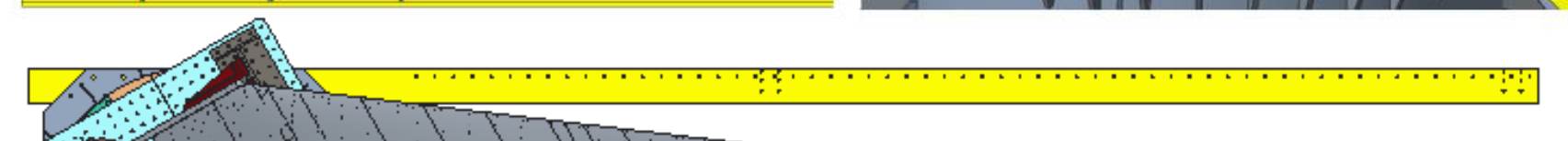
Reaction Plates 


\section{Test Setup - GVT Test Setup, Simplified Actual Testing}

- Simplified actual testing setup: Performed GVT with WLTF table on FLL floor

- Simplified GVT shaker setup since the wingtip is $\approx 50$ " off the floor, rather than the wingtip being 124" high

- Boundary conditions: WLTF table on FLL floor supported by four retractable feet \& one location on the table that was secured to the FLL floor tracks with a strap

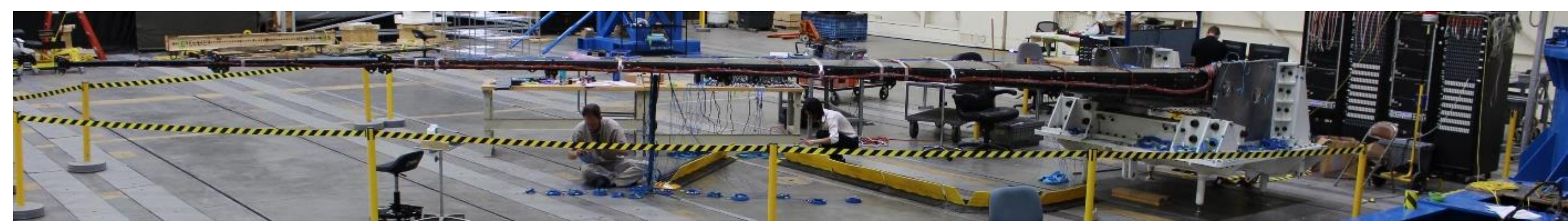

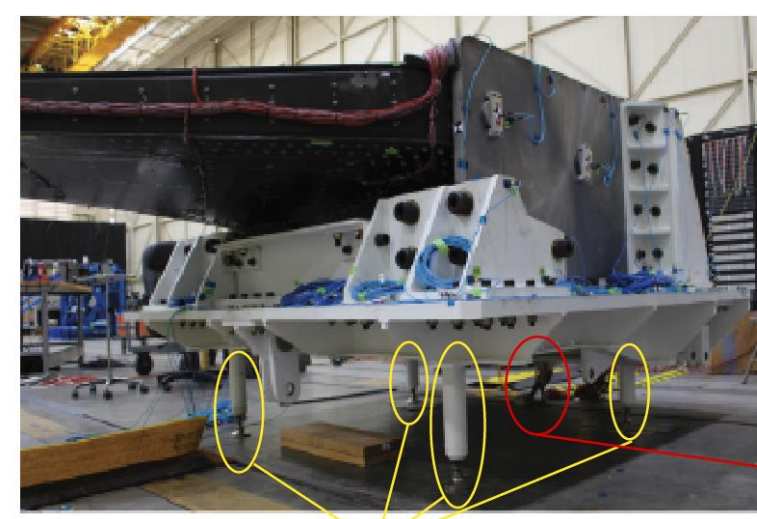

Four retractable feet boundary condition

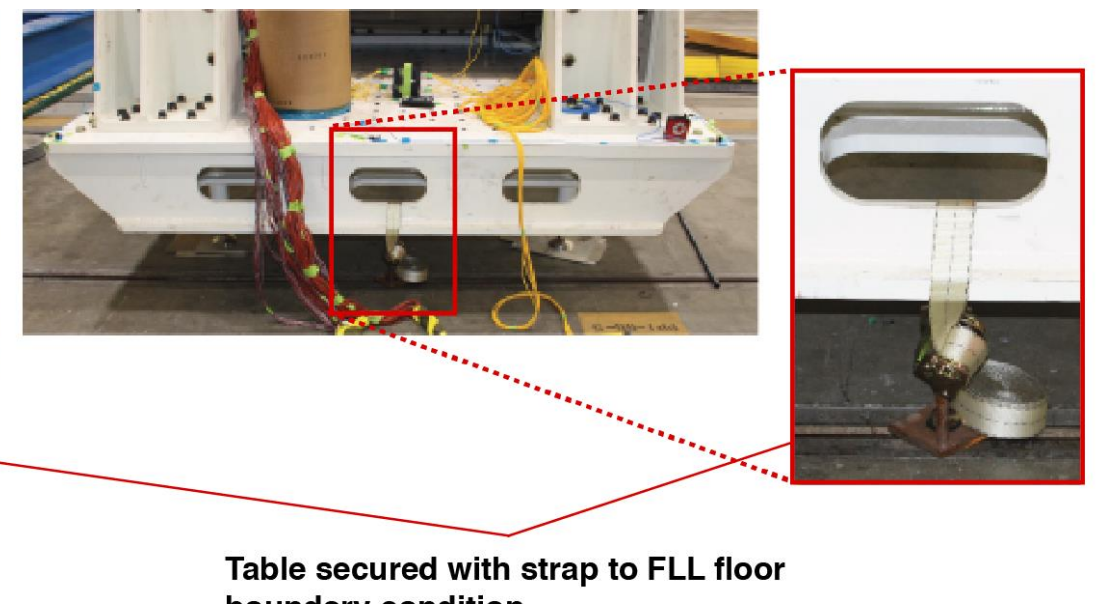

Table secured with strap to FLL floor boundary condition
WLTF Table Boundary Condition on FLL Floor (NOT ideal for traditional modal testing) 


\section{Test Setup - GVT Equipment}

\section{- GVT Equipment}

- Accelerometers

- PCB T333B32 uniaxial accels

- PCB T356A16 triaxial accels

- PCB 393B04 seismic uniaxial accels

- Excitation Systems

- Shakers: MB Dynamics Electromagnetic Modal 110 shaker

- Data Acquisition (DAQ) system: Brüel \& Kjær LAN-XI DAQ

- DAQ capable of recording 328 channels

- Mainframes

- LAN-XI 5-slot Main frame, 2 qty

- Modules

- LAN-XI 11-slot Main frame, 2 qty

- LAN-XI 4ch input + 2ch output 3160 source modules, 7 qty

- Capable of running 14 shakers

- Capable of recording 28 channels

- LAN-XI 12-channel 3053 modules, 25 qty

- GVT Software:

- Capable of recording 300 channels

- Ideas Test (acquired time histories)

- IMAT (all test related analysis \& FBC analysis)

Note: Some GVT hardware was provided by Contractor

Brüel \& Kjær LAN-XI DAQ

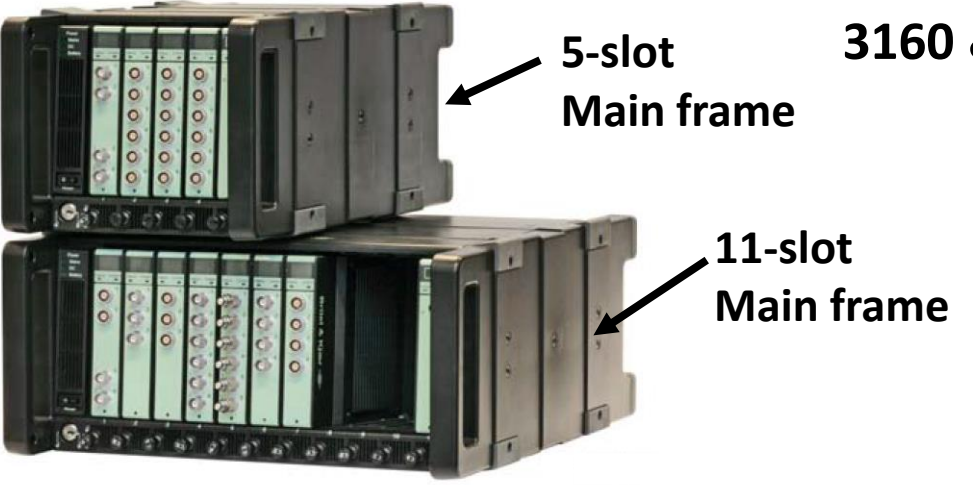

PCB 393B04

Seismic Uniaxial Accel

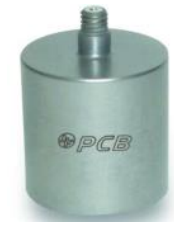

MB Modal 110

Shaker

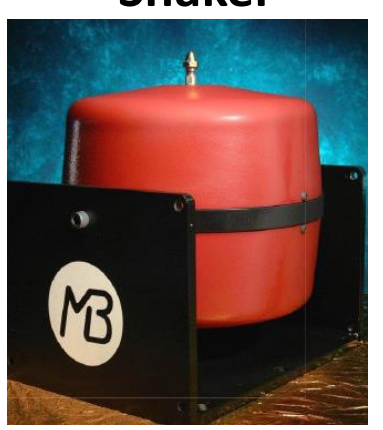




\section{Test Setup - LAN-XI DAQ}

- LAN-XI DAQ frontend setup: Four mainframes (two 5-slot \& two 11-slot) capable of driving 14 shakers \& recording 328 channels with network switch daisy chaining modules

- MF\#1: five source module (3160)

- MF\#2: two source modules (3160) \& three 12-channel input module (3053)

- MF\#2: eleven 12-channel input modules (3053)

- MF\#2: eleven 12-channel input modules (3053)

LAN-XI DAQ Setup for PAT Wing GVT

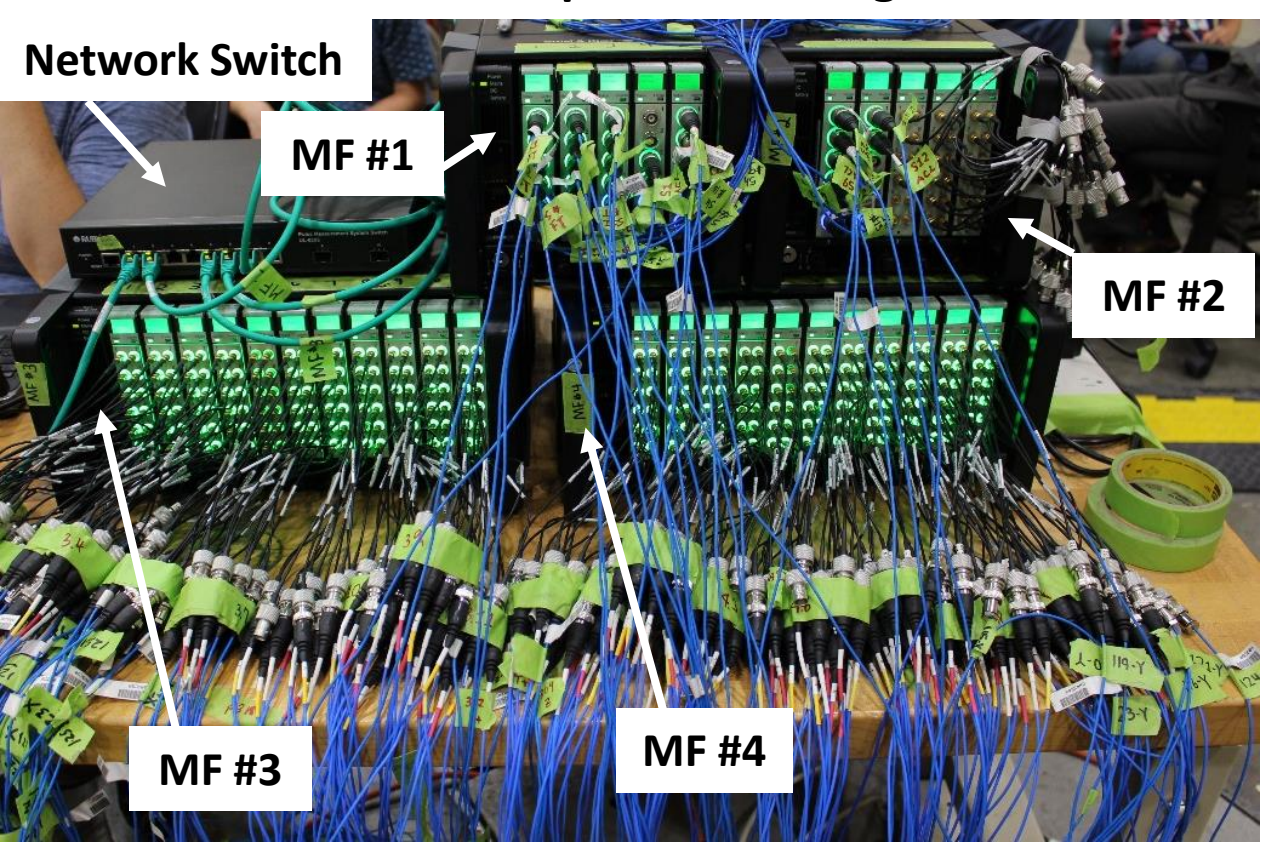

Total: 288 Channels Enabled (Accels \& Force Transducers)

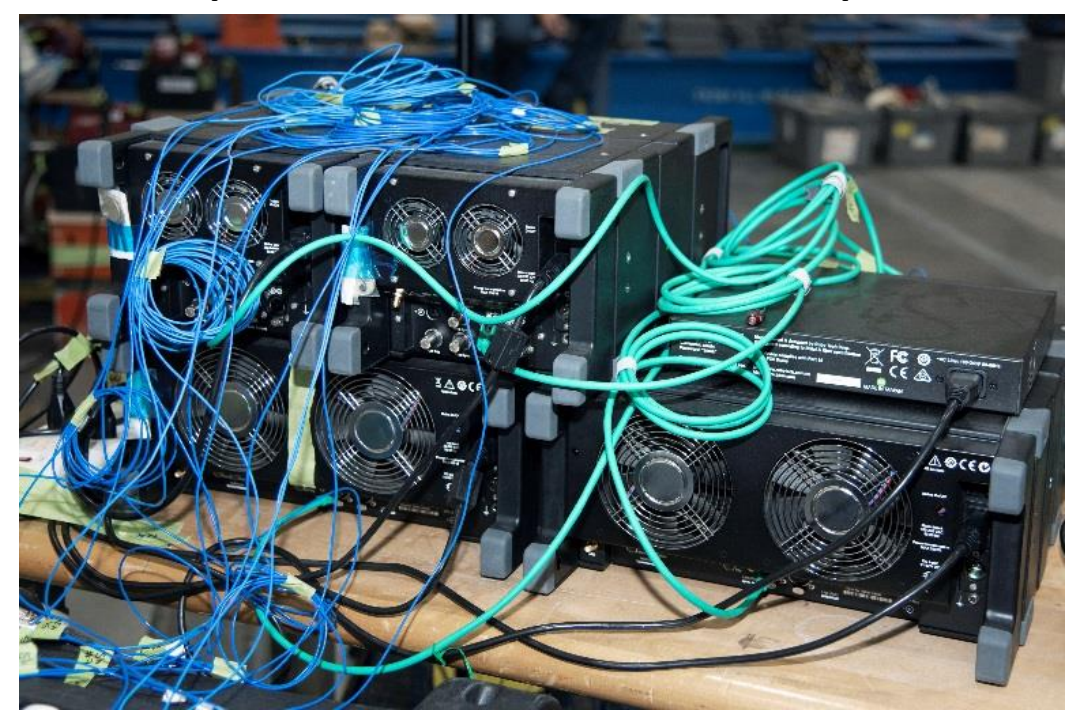

Note: Some LAN-XI source modules were provided by Contractor 


\section{Test Setup - Accel Wing Photos}

- Accel coordinates obtained from FEM

- All nodes in global coordinate system wrt WLTF

- X+ (out Trailing Edge), Y+ (out Outboard), Z+ (up)

- Used $30^{\circ}$ template to install wing accels with correct angle orientation
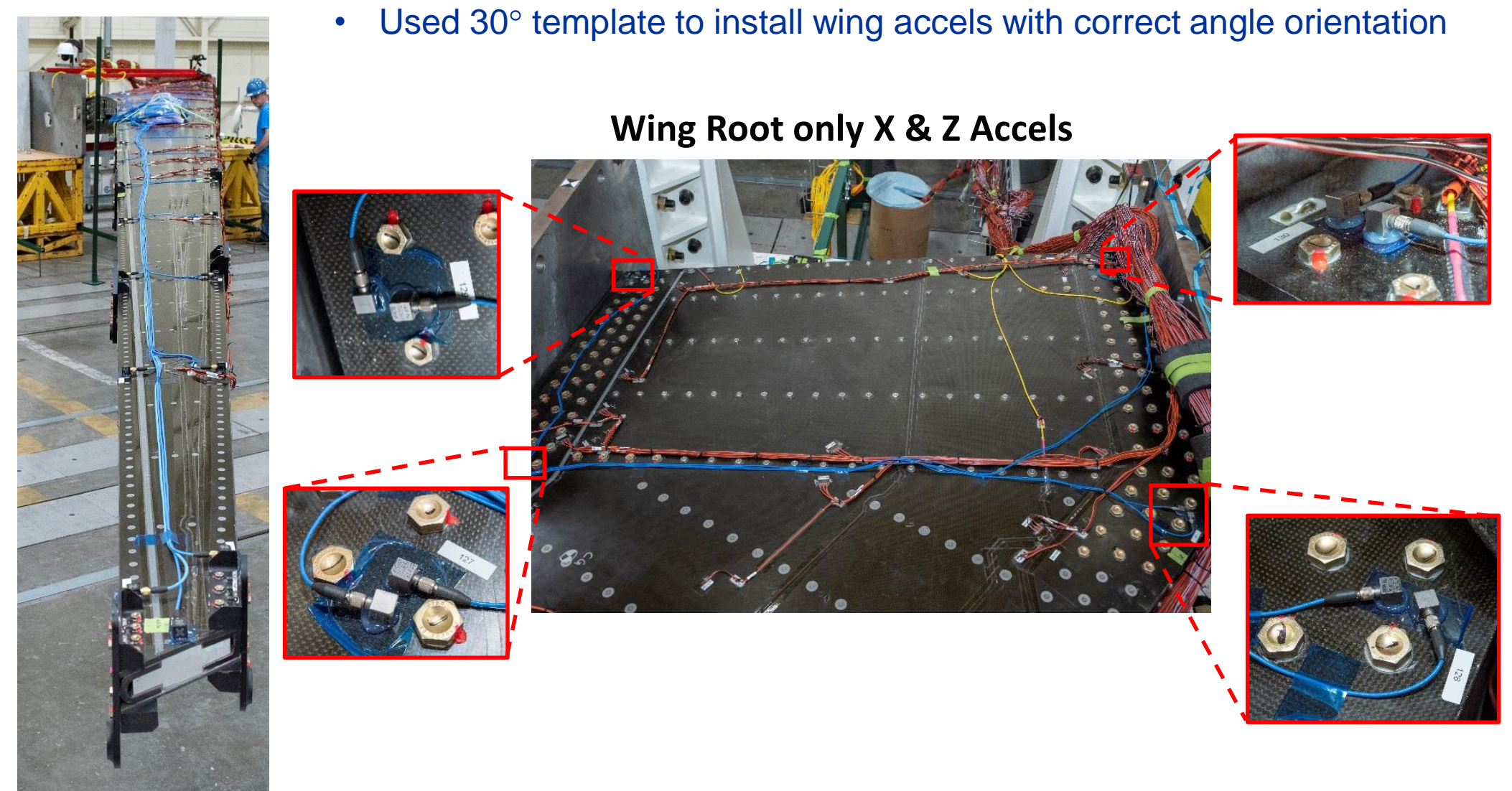

Wingtip Triaxial Accels
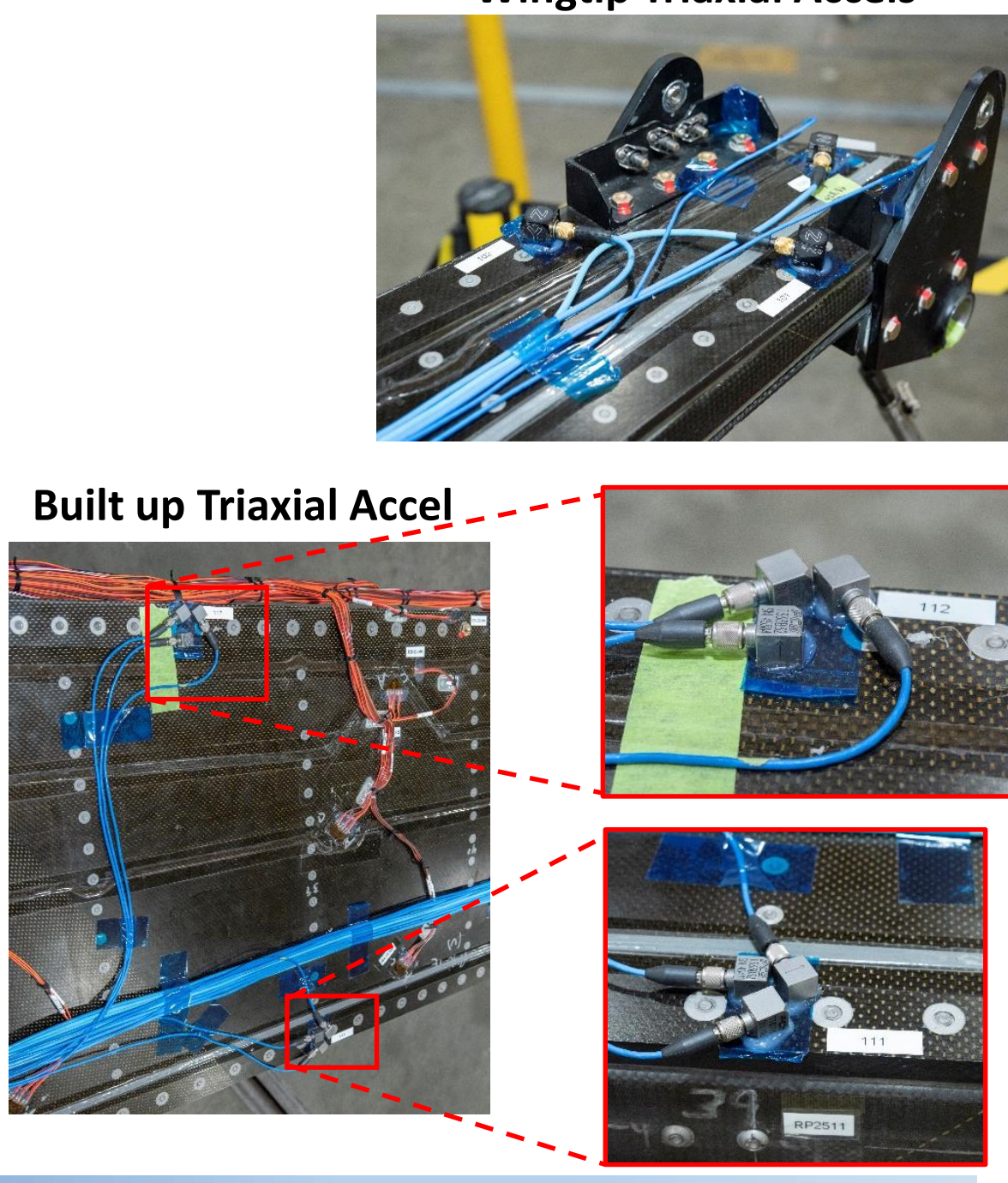


\section{Test Setup - Accel Attachment Hardware Photos}

- Some attachment hardware accels were installed before wing was installed on WLTF table

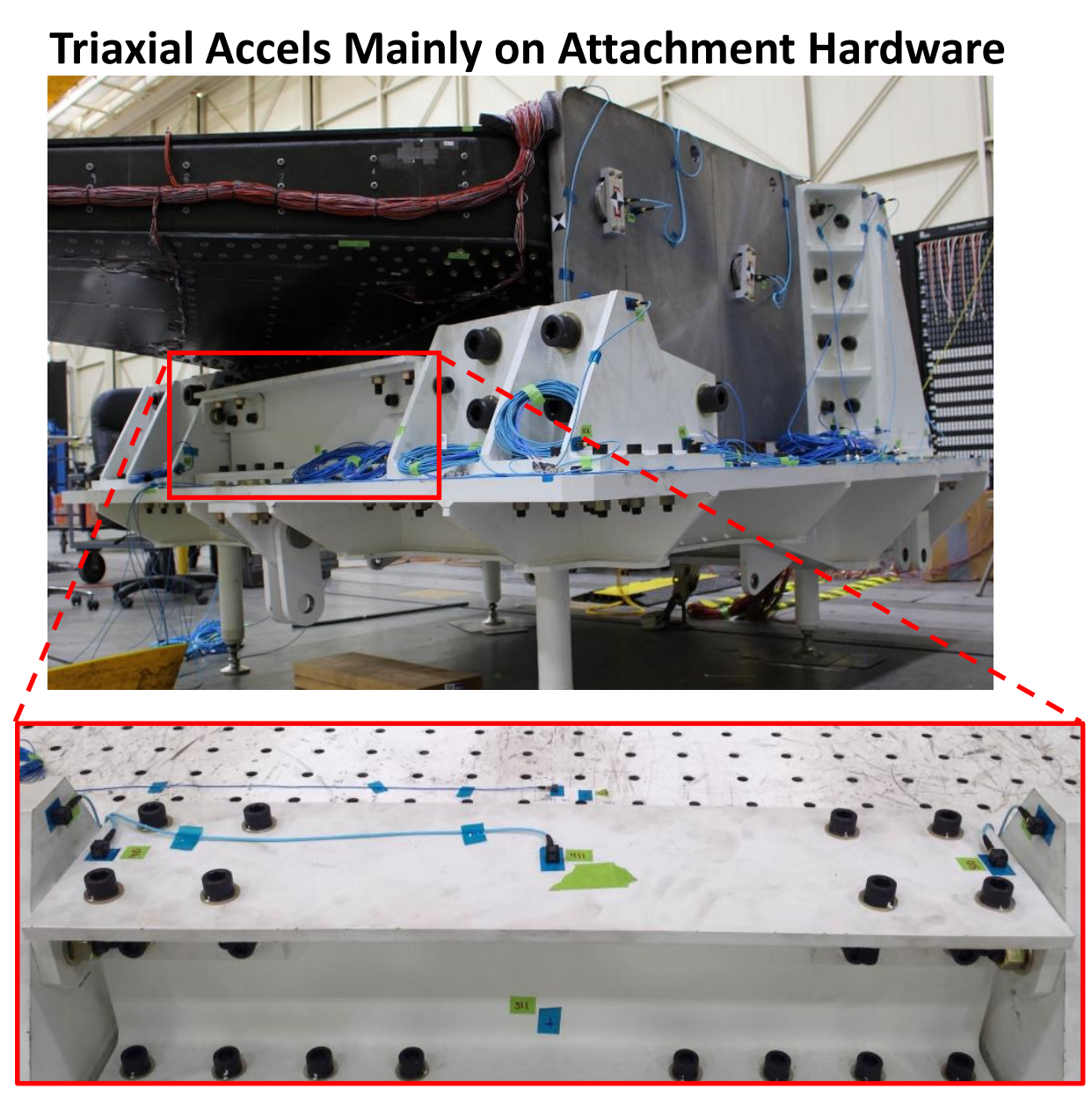

Attachment Hardware Accels - Leading Edge side

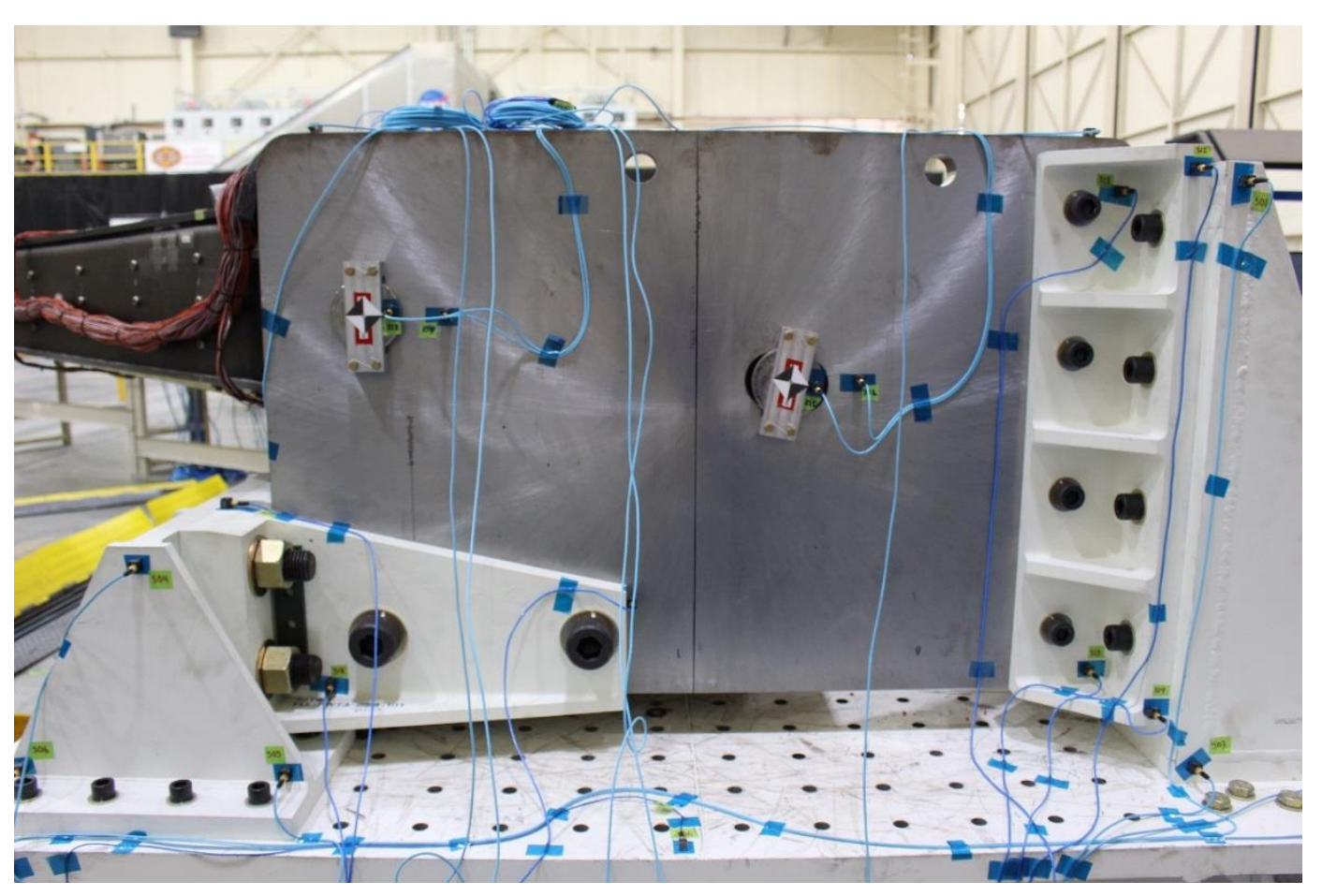




\section{Test Setup - Shaker Force Transducer \& Accel Photos}

- Wingtip shaker - Force Transducers \& Accels $(100 \mathrm{mV} / \mathrm{g})$

- "Fixed" shakers on Table \& Attachment Hardware - Force Transducers \& Seismic Accels (1000 mV/g)

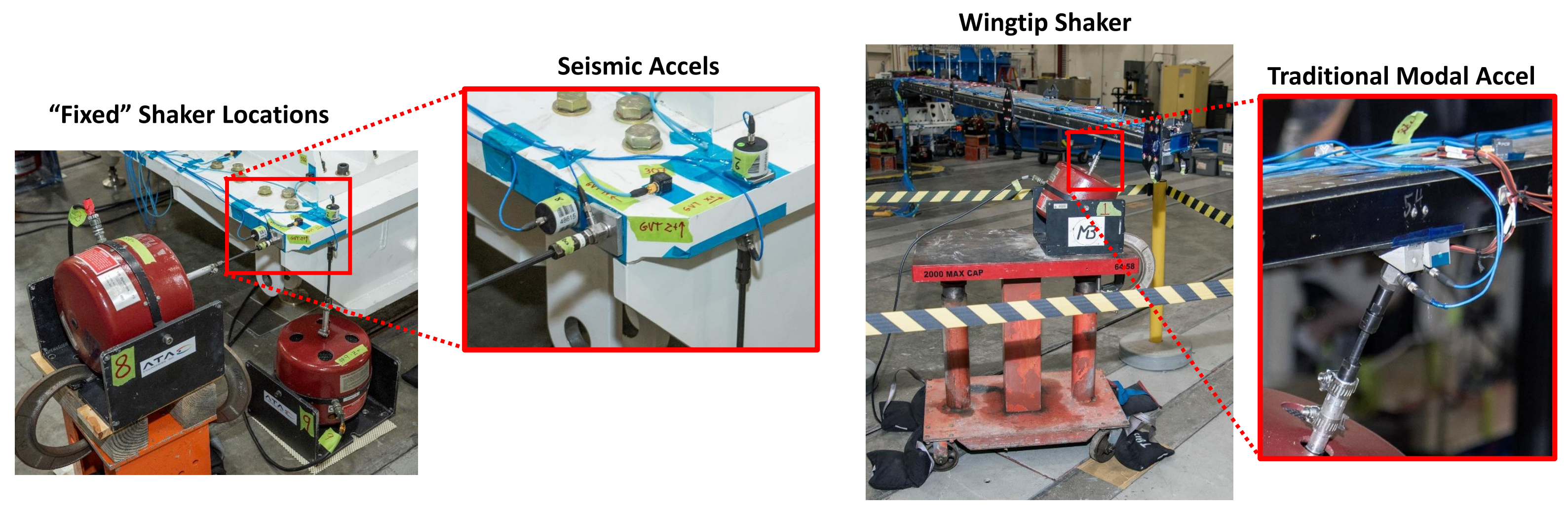




\section{GVT Shaker Layout - Fixed Base Correction Method}

- FBC method requires multiple independent drive points (shakers) mounted to test fixture \& test article

- Shaker layout depends on where FBC technique is trying to fix the BC

- Needs at least as many independent sources as there are independent boundary deformations of the desired fixed hardware in the test article frequency range of interest

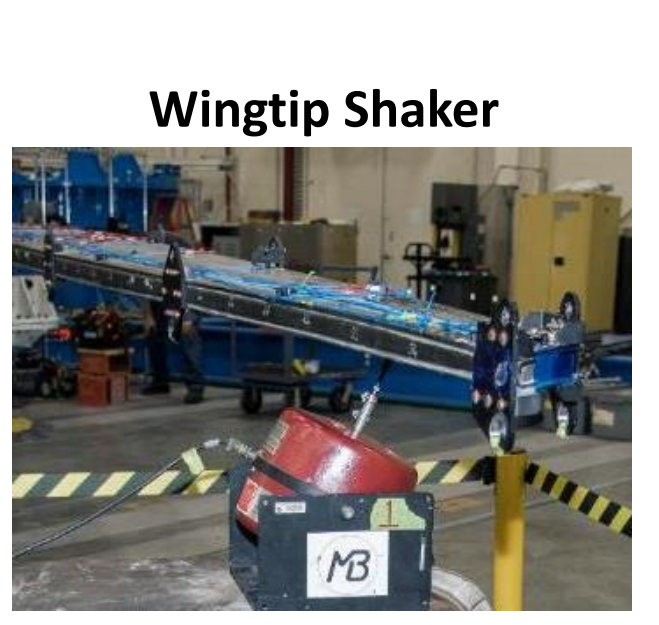

"Fixed" WLTF Shaker Locations

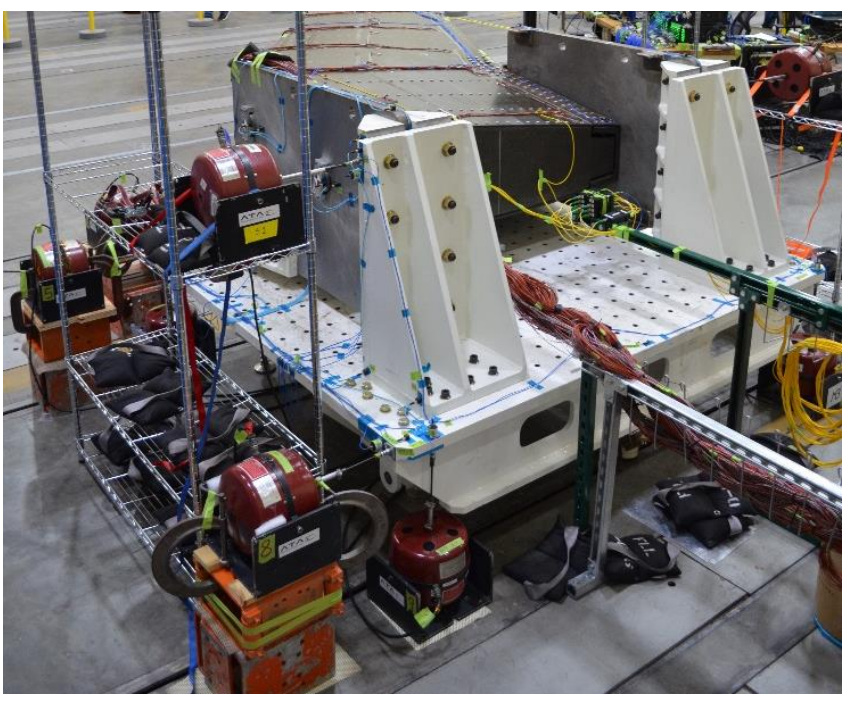

- Shaker placement around the WLTF was adjusted to excite primary base modes \& maximize the capability of the FBC to decouple the base modes from the wing modes

- Higher shaker forces were required on the base

- A few different shaker configurations were attempted to find optimal shaker configuration which fixed the reaction table

- Shaker direction on reaction table is important \& eliminates the effect of the reaction table from moving in the shaker direction

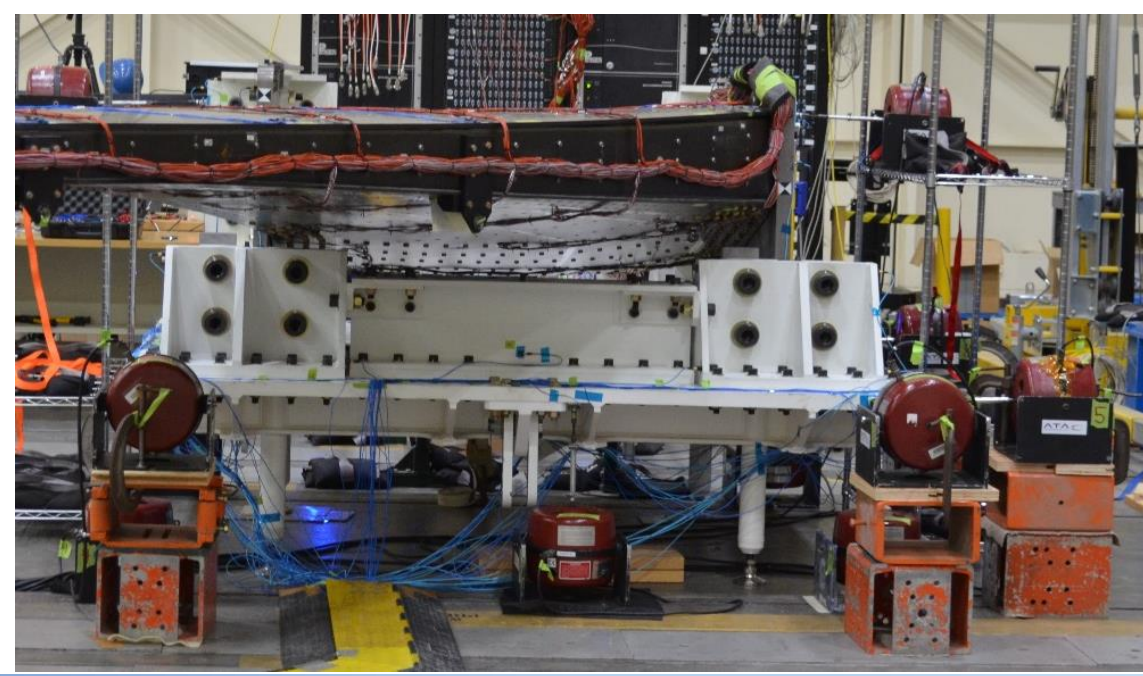




\section{PAT Wing GVT Shaker Layouts for FBC}

- Shaker configurations for FBC method

- 10 Shakers, Initial Pass -9 on reaction table, 1 on wingtip

- 12 Shakers, Second Pass - Added 2 on aft triangular brackets (fore/aft)

- 14 Shakers, Final Pass - Added 2 on wing root reaction plates (fore/aft)

PAT Wing GVT - Shaker Configurations

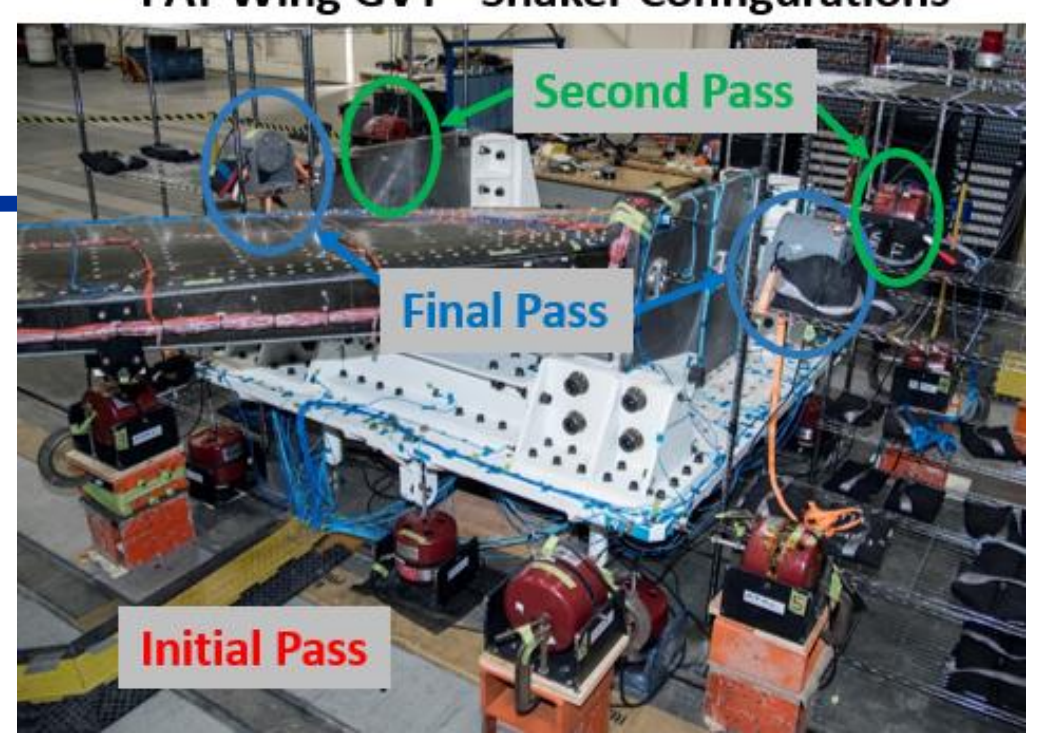

10 Shakers, Initial Pass

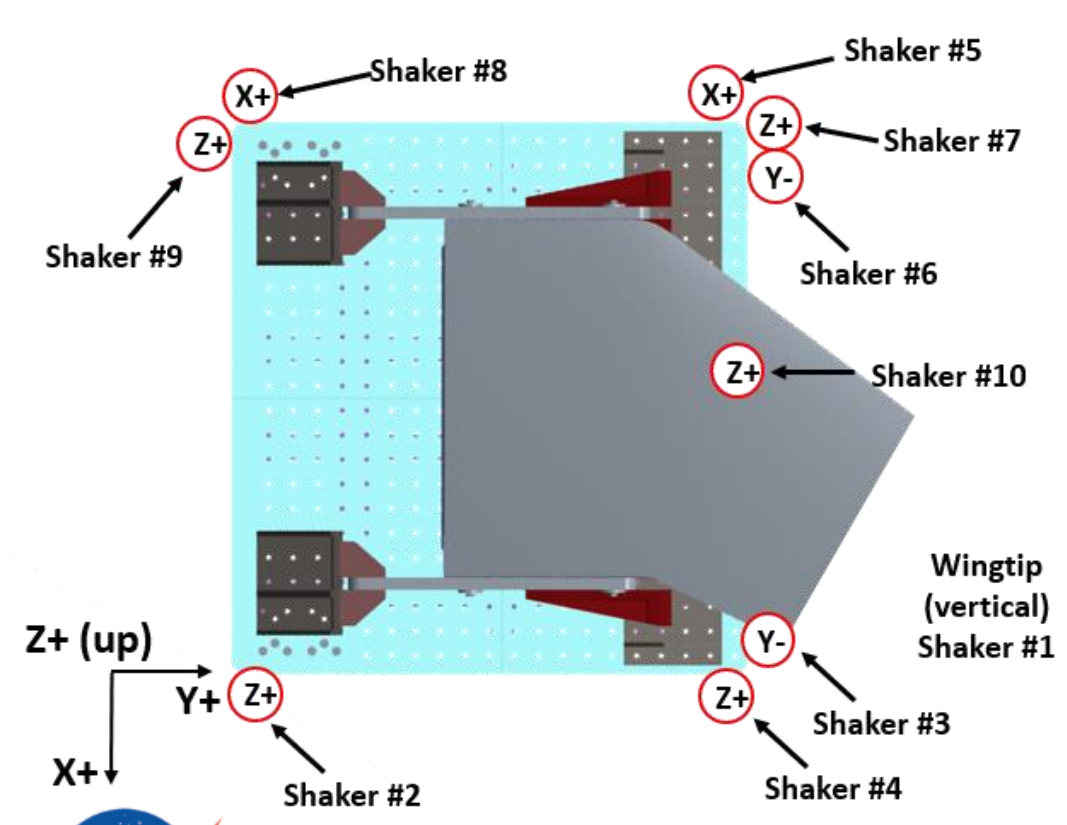

12 Shakers, Second Pass

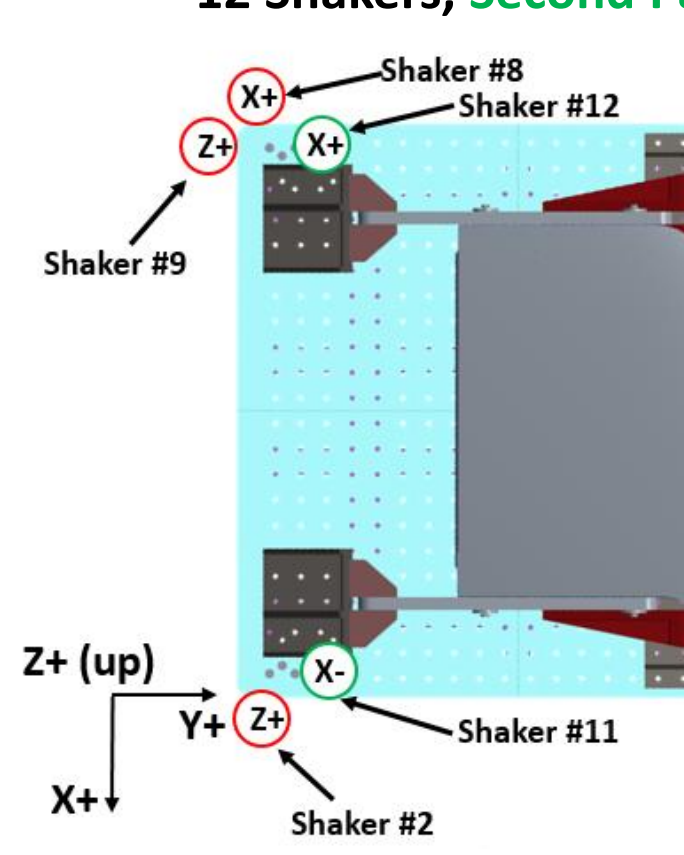

\section{Shakers, Final Pass}

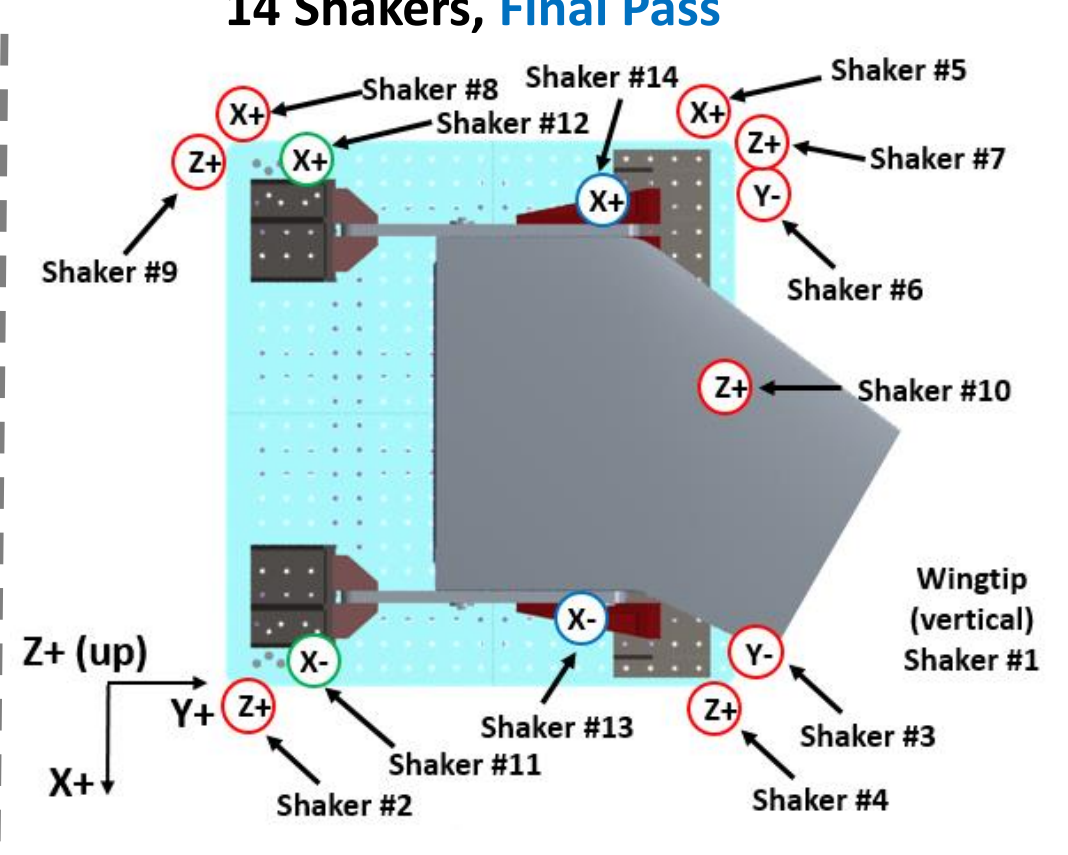




\section{PAT Wing GVT Shaker Layouts \& FEM Boundary Conditions}

- FEM "Fixed" boundary conditions were applied to all nodes on related hardware

- 10 Shakers, Initial Pass - 9 on reaction table, 1 on wingtip

- 12 Shakers, Second Pass - Added 2 on aft triangular brackets (fore/aft)

- 14 Shakers, Final Pass - Added 2 on wing root reaction plates (fore/aft)

10 Shakers, Initial Pass Reaction Table "Fixed"

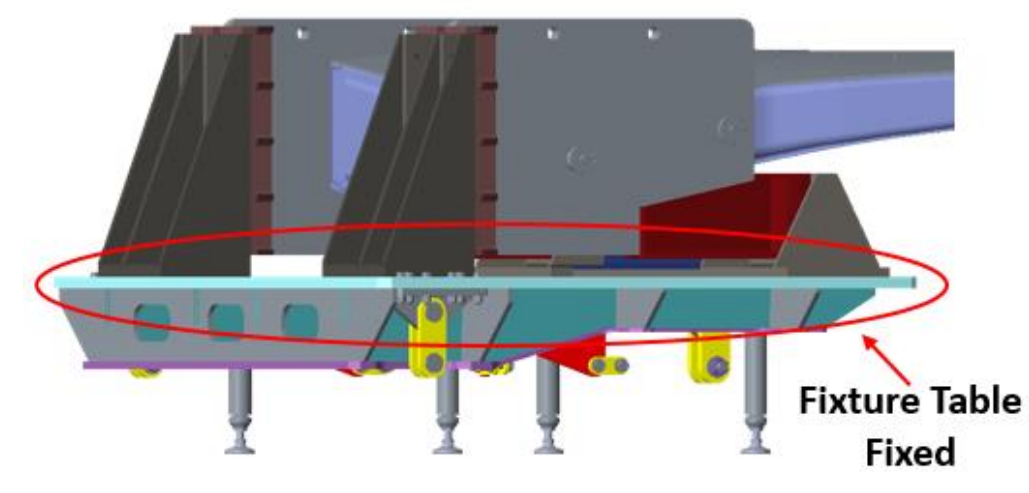

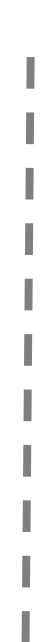

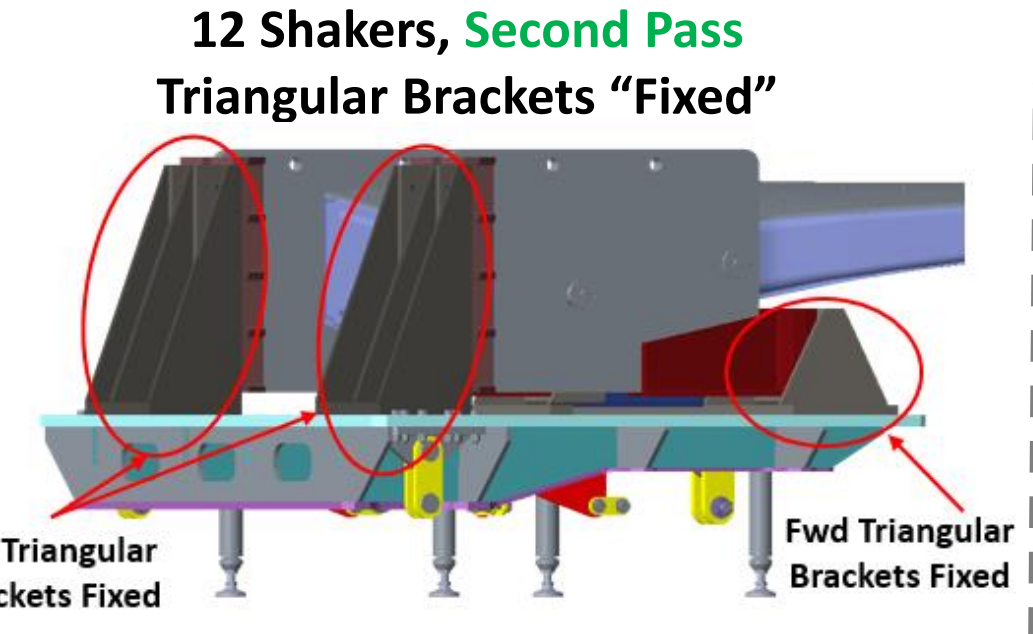

FEM “Fixed" Boundary Conditions
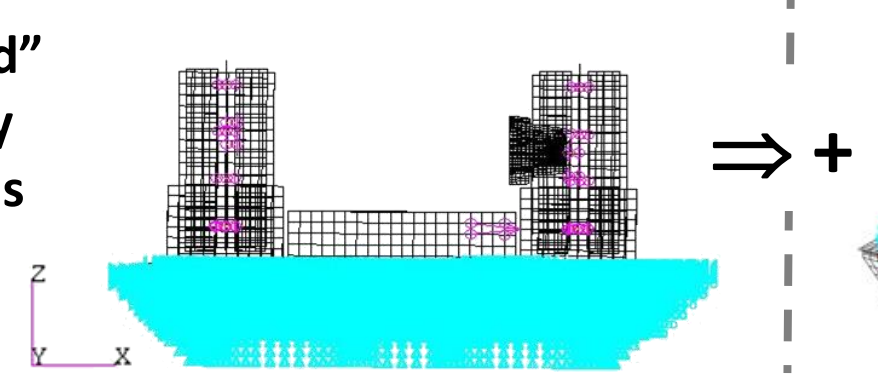

14 Shakers, Final Pass Everything "Fixed", but Wing

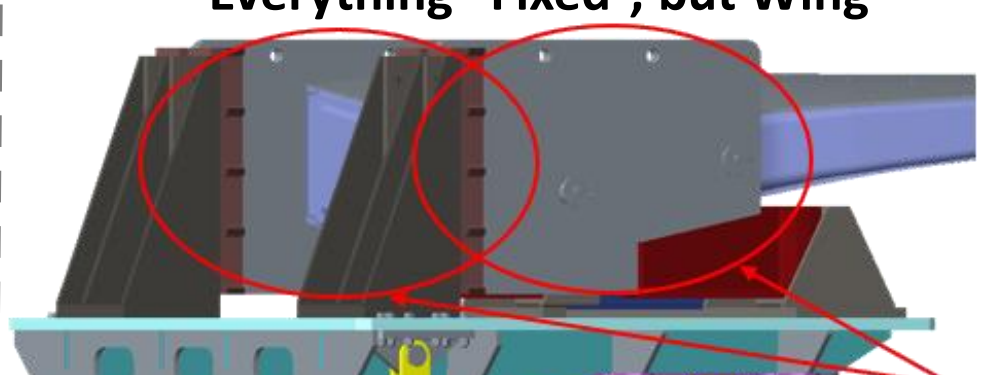$$
\text { II }
$$

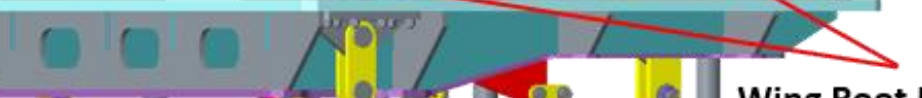

Wing Root Reaction Plates Fixed

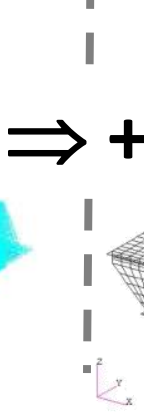




\section{Results - 14 Shakers, Uncorrected vs. FBC}

- FBC mode shapes show very little base deflection

- Uncorrected mode shapes show significant base rotation

- Wing bending modes coupled the least with WLTF (setup is stiffer vertically than in other directions)

- Wing fore/aft modes coupled the most with WLTF \& required significant correction

- FBC method was able to remove a majority of the dynamics of the static test fixture to acquire fixed base modes while still accurately measuring the shape of the wing

- Promising sign of the effectiveness of the FBC method

\section{Frequency \% Difference to FEM}

14 Shakers GVT: Uncorrected vs. Fixed Base Correction

\begin{tabular}{|c|c|c|c|c|c|c|}
\hline \multirow{2}{*}{$\begin{array}{c}\text { Mode } \\
\#\end{array}$} & $\begin{array}{c}\text { Mode } \\
\text { Description }\end{array}$ & FEM & $\begin{array}{c}\text { 14-Shaker } \\
\text { Uncorrected }\end{array}$ & $\begin{array}{c}\text { 14-Shaker } \\
\text { FBC }\end{array}$ & $\begin{array}{c}\text { 14-Shaker } \\
\text { Uncorrected }\end{array}$ & $\begin{array}{c}\text { 14-Shaker } \\
\text { FBC }\end{array}$ \\
\cline { 3 - 7 } & W1B & 3.4 & 3.5 & 3.6 & $3 \%$ & $5 \%$ \\
\hline 2 & W2B & 10.4 & 10.1 & 10.0 & $-3 \%$ & $-4 \%$ \\
\hline 3 & W1F/A & 11.3 & 5.1 & 11.0 & $-55 \%$ & $-3 \%$ \\
\hline 4 & W3B & 22.5 & 22.0 & 21.2 & $-2 \%$ & $-6 \%$ \\
\hline 5 & W2F/A & 31.7 & 16.5 & 30.2 & $-48 \%$ & $-5 \%$ \\
\hline 6 & W4B & 37.2 & 35.4 & 35.2 & $-5 \%$ & $-5 \%$ \\
\hline 7 & W5B (W1T) & 51.8 & 50.4 & 52.2 & $-3 \%$ & $1 \%$ \\
\hline 8 & W1T & 55.2 & 56.5 & 56.4 & $2 \%$ & $2 \%$ \\
\hline
\end{tabular}

14 Shaker Test Results - Wing $2^{\text {nd }}$ Fore/Aft GVT: Uncorrected vs. Fixed Base Correction
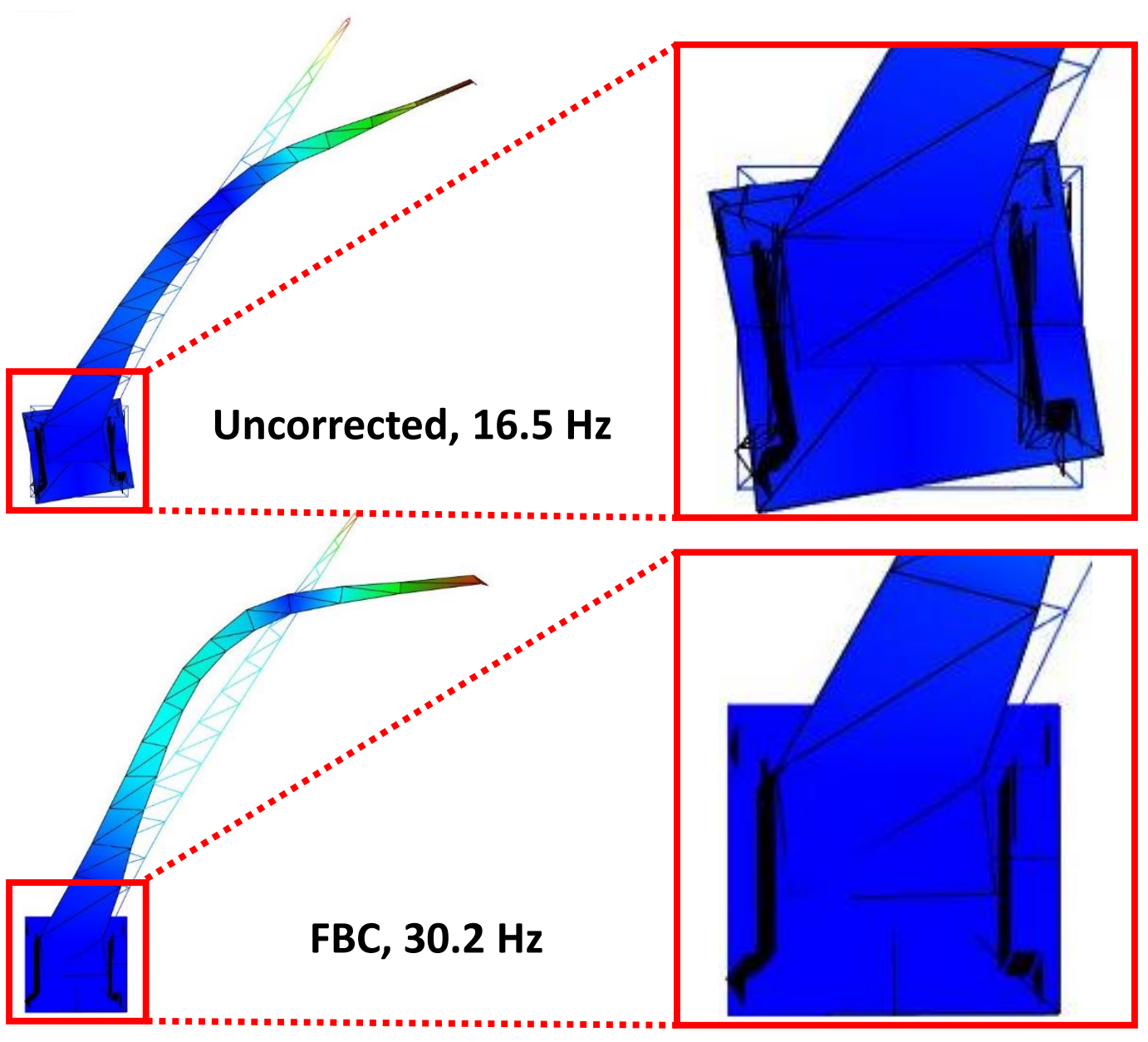


\section{Results - 14 Shakers, Uncorrected vs. FBC}

- Modal Assurance Criteria (MAC) cleans up when applying FBC

- Uncorrected modes have substantial base rotation

- FBC eliminates some modes when fixing the base

Modal Assurance Criteria (MAC), 14 Shaker Tests

\section{Uncorrected vs. FEM}

Uncorrected

14 Shakers

Fore/Aft Wingtip Excitation

FEM/Test Cross MAC Table

FEM Shapes

\begin{tabular}{|l|r|r|r|r|r|r|r|r|r|}
\hline \multirow{2}{*}{ Fully Fixed Pretest FEM (Not Updated) } & 1 & 2 & 3 & 4 & 5 & 6 & 7 & W1B \\
\cline { 2 - 9 } & W2B & W1F/A & W3B & W2F/A & W4B & W5B (W1T) & W1T (W5B) \\
\hline
\end{tabular}

\begin{tabular}{|c|c|c|c|c|c|c|c|c|c|c|}
\hline & & MAC & 3.4 & 10.4 & 11.3 & 22.5 & 31.7 & 37.2 & 51.8 & 55.2 \\
\hline & W1B & 3.5 & 0.99 & 0.30 & & 0.16 & & & & \\
\hline & W1F/A (Base) & 5.1 & & & 0.83 & & & & & \\
\hline 3 & W2B (W1F/A, Base) & 9.1 & 0.26 & 0.50 & 0.34 & 0.17 & & & & \\
\hline 4 & W2B & 10.1 & 0.32 & 0.98 & & 0.40 & & 0.19 & & \\
\hline 5 & W2F/A (Base) & 16.5 & & & 0.87 & & 0.53 & & & \\
\hline 6 & W3B (W2F/A, Base) & 20.2 & & 0.31 & & 0.73 & & 0.37 & & \\
\hline & W3B (Base) & 22.0 & & 0.28 & & 0.88 & & 0.35 & & \\
\hline & W2F/A (W4B, Base) & 34.1 & & & 0.20 & 0.15 & 0.66 & 0.26 & & 0.21 \\
\hline & W4B (W2F/A, Base) & 35.4 & & & & 0.18 & 0.30 & 0.71 & & \\
\hline 10 & W5B (W1T, Base) & 50.4 & & & & & & 0.26 & 0.23 & 0.35 \\
\hline & W1T (Base) & 56.5 & & & & & & & 0.70 & 0.30 \\
\hline
\end{tabular}

Note: Duplicated modes with lots of base motion eliminated when applying FBC

\section{Fixed Base Correction vs. FEM}

Fixed Base Corrected $\quad$ FEM/Test Cross MAC Table

Fore/Aft and Vertical Wingtip Excitation Fully Fixed Pretest FEM (Not Updated)

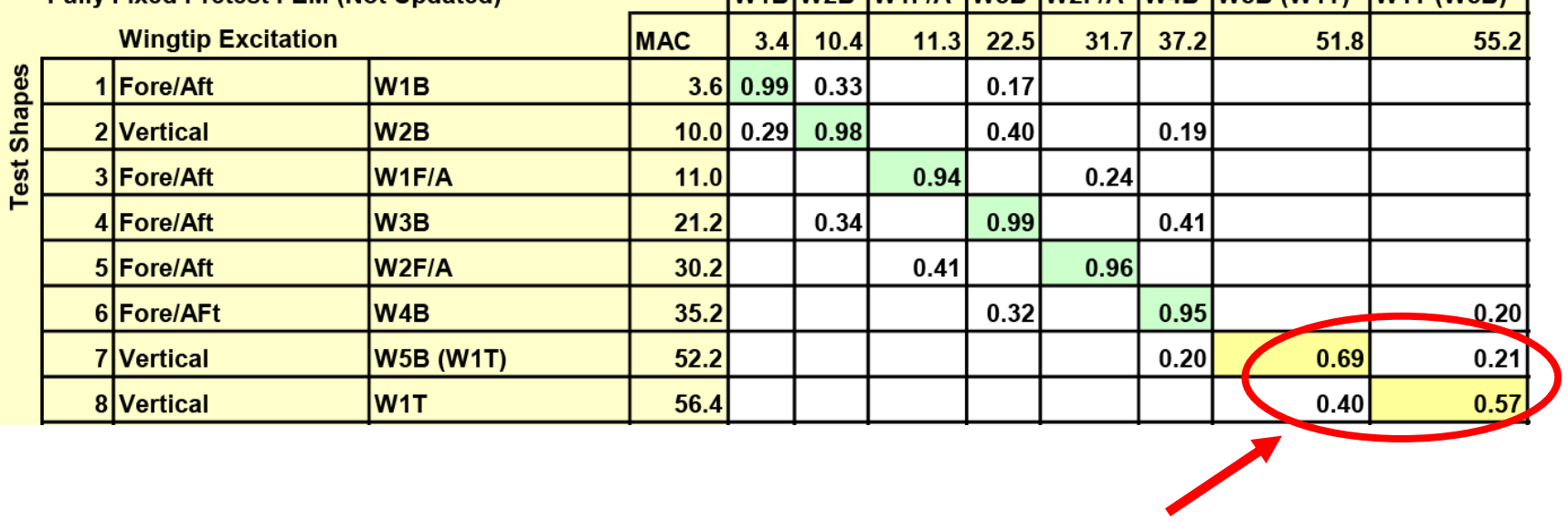

Note: FEM has W5B \& W1T highly coupled where GVT showed wing is not as coupled 


\section{Summary}

- PAT Wing GVT results show success and the feasibility of using the Fixed Base Correction (FBC) method to decouple the wing \& test fixture modes for a flexible wing mounted to a dynamically active static test fixture

- Fixed Base Correction method

- FBC results produce test results with reliable boundary conditions to replicate in analytical models

- FBC has the potential to change how some modal testing is traditionally performed and can save money and schedule time by eliminating an independent setup for modal testing

- Many potential scenarios where this technique can be used on future tests of structures mounted on other dynamically active test fixtures 


\section{Questions}

